\title{
Intoxications au dextropropoxyphène et au tramadol : bilan d'un an de recueil
}

\section{Dextropropoxyphene and tramadol intoxica- tions: one year assessment of collection}

Sabine COHEN ${ }^{(1) *}$, Monique MANCHON ${ }^{(1)}$, Michèle ARTUR ${ }^{(2)}$, Bernard CAPOLAGHI ${ }^{(3)}$, Raphaël DENOOZ ${ }^{(4)}$, Nicole HOUDRET ${ }^{(5)}$, Nicole JOURDIL ${ }^{(\oplus)}$, Marie-France KERGUERIS ${ }^{(7)}$, Denis LAMIABLE $^{(8)}$, Mustapha MOULSMA ${ }^{(9)}$, Nouredine SADEG ${ }^{(10)}$, Alain TURCANT ${ }^{(1)}$ (Groupe de travail "Toxicologie hospitalière" de la SFTA)

(1) Laboratoire des Urgences Biochimiques et Toxicologiques, CHU Lyon-Sud

(2) Laboratoire de Pharmacologie et Toxicologie, CHU Dijon

(3) Laboratoire de Biologie, CH Thionville

(4) Laboratoire Toxi-Clinique, CHU Liège

(5) Laboratoire de Biochimie, CHU Lille (6) UF Pharmacologie-Département de Biologie et Pathologie de la Cellule, CHU Grenoble

(7) Laboratoire de Pharmacologie-Toxicologie, CHU Nantes

(8) Laboratoire de Pharmacologie-Toxicologie, CHU Reims (9) UF Pharmacologie-Analyse des Éléments trace, CHU Lyon (10) Laboratoire Claude Bernard, CH Pontoise (11) Laboratoire de Pharmacologie, CHU Angers

*Auteur à qui adresser la correspondance : Sabine COHEN, Laboratoire des Urgences Biochimiques et Toxicologiques, Centre Hospitalier Lyon Sud, 165, Chemin du Grand Revoyet - 69495 PIERRE-BENITE Cedex Tél : 04788612 49/37 - Fax : 0478862198 - E-mail : sabine.cohen@chu-lyon.fr 


\section{RÉSUMÉ}

La prescription de plus en plus fréquente du dextropropoxyphène $(D P)$ et du tramadol, deux antalgiques opiö̈des, a conduit les membres du groupe de travail «Toxicologie hospitalière » de la Société Française de Toxicologie Analytique (SFTA) à étudier leur toxicité clinique observée. L'étude a consisté en l'analyse des cas où l'une ou l'autre de ces molécules a été identifiée au décours d'un screening toxicologique réalisé durant l'année 2004 dans les laboratoires hospitaliers des membres de ce groupe. Pour chaque patient ont été recueillis les données démographiques, les concentrations plasmatiques en dextropropoxyphène, norpropoxyphène (NP) ou tramadol, la concentration plasmatique en paracétamol, les médicaments associés et les signes cliniques (digestifs, respiratoires, cardiaques et neurologiques). Aucune relation entre les concentrations plasmatiques de dextropropoxyphène et de paracétamol, ni entre celles de dextropropoxyphène ou de tramadol et la gravité clinique n'a été observée. Les concentrations plasmatiques observées en dextropropoxyphène sont faibles mais doivent attirer l'attention sur une éventuelle intoxication au paracétamol. Les concentrations plasmatiques en tramadol peuvent être élevées mais les intoxications sont dans l'ensemble peu sévères et les concentrations toxiques semblent très supérieures à la concentration de $1 \mathrm{mg} / \mathrm{L}$.

\section{MOTS-CLÉS}

Dextropropoxyphène, tramadol, toxicité, paracétamol.

\section{Introduction}

L'étude présentée a été menée par les membres du groupe de travail «Toxicologie hospitalière » de la SFTA sur dix sites en France et un en Belgique. Le dextropropoxyphène et le tramadol sont deux antalgiques opioïdes entrant dans la composition de nombreuses spécialités pharmaceutiques. En France, 17 spécialités contiennent du dextropropoxyphène et $44 \mathrm{du}$ tramadol (1). Le dextropropoxyphène seul n'est plus commercialisé. Il est systématiquement associé au paracétamol. Le tramadol est le plus fréquemment commercialisé seul et est associé au paracétamol dans deux spécialités : Zaldiar ${ }^{\circledast}$ et Ixprim $^{\circledast}$. En Belgique, il existe encore une spécialité qui ne contient que du dextropropoxyphène $\left(\right.$ Depronal $\left.^{\circledR}\right)$, les autres spécialités associent le dextropropoxyphène au paracétamol. Actuellement, ces deux antalgiques sont très largement prescrits. En effet, d'après les données de l'AFSSAPS (Agence française de sécurité sanitaire des produits de santé), en 2003 le Propofan ${ }^{\circledR}$ (paracetamol-caféine-dextropropoxyphène) et le Di-antalvic ${ }^{\circledR}$ (paracétamol-dextropropoxyphène) étaient respectivement aux $8^{\text {ème }}$ et $9^{\text {ème }}$ rangs des spécialités les plus vendues en officine en France (1). Le Topalgic ${ }^{\circledR}$ (tramadol) n'était pas présent parmi les 50 spécialités les plus vendues, mais en 2003 le tramadol était moins prescrit qu'aujourd'hui et les spécia-

\section{SUMMARY}

Dextropropoxyphene and tramadol are two opioid analgesics that are prescribed more and more often. So clinical toxicology members of SFTA proposed to study the clinical toxicity of these molecules. For one year, the committee members have collected all cases of toxicological screening in which dextropropoxyphene or tramadol were identified. We analysed the demographic data, the dextropropoxyphene and norpropoxyphene or tramadol plasma concentrations, the acetaminophen plasma concentration, the co medications, and the adverse events (digestive, neurological, pulmonary, heart). No relation between dextropropoxyphène and acetaminophen plasma concentrations has been observed; no relation between dextropropoxyphene or tramadol plasma concentrations and the seriousness of the intoxication has been observed either. Plasma dextropropoxyphene concentrations are low but dextropropoxyphene or norpropoxyphene identification in plasma should predict possible acetaminophen intoxication. Plasma tramadol concentrations can be high but intoxications are often benign and toxic plasma concentrations seem to be more than $1 \mathrm{mg} / \mathrm{L}$.

\section{KEY-WORDS}

Dextropropoxyphene, tramadol, toxicity, acetaminophen.

lités associant tramadol et paracétamol venaient juste d'être commercialisées (1). Afin de voir l'implication de ces deux molécules dans les intoxications médicamenteuses volontaires et de faire le point sur leur toxicité clinique observée, les membres du groupe de travail ont recueilli sur une année les cas d'intoxications pour lesquelles il y avait une participation de dextropropoxyphène ou de tramadol.

\section{Matériels et méthodes}

Nous avons recueilli et analysé les dossiers cliniques des cas où une mise en évidence de dextropropoxyphène ou de tramadol, dans le sang ou les urines, a été observée au décours d'un screening toxicologique pratiqué au cours de l'année 2004 dans les laboratoires hospitaliers qui ont participé à l'étude. Ont été exclus les dossiers rapportant l'utilisation de l'une ou l'autre de ces molécules dans un but de toxicomanie. Les screening ont été réalisées par chromatographie liquide haute performance couplée à une détection UV en barrettes de diodes ou à réseau tournant. Il n'y a pas de standardisation des méthodes analytiques, chaque site ayant utilisé les techniques d'extraction et de screening validées au sein de son laboratoire. Les techniques de screening utilisées ainsi que les méthodes de dosage du dextropropoxyphène, norpropoxyphène, tramadol et 
celle du paracétamol sont précisées par le Tableau I. Nous avons étudié les données démographiques, les concentrations plasmatiques en dextropropoxyphène, norpropoxyphène (métabolite actif du dextropropoxyphène), tramadol (le métabolite actif du tramadol, le Odesméthyl tramadol, n'a pas été quantifié), la concentration en paracétamol, les médicaments associés retrouvés et les signes cliniques : digestifs, respiratoires, cardiaques, neurologiques. Les concentrations plasmatiques des médicaments associés n'ont pas été recueillies.

\section{Résultats}

\section{La population :}

Le tableau I montre que tous les sites n'ont pas répondu à la fois pour le dextropropoxyphène et pour le tramadol. Sur l'ensemble des screening réalisés ont été répertoriés 180 cas avec une participation de dextropropoxyphène et 116 cas avec du tramadol. Ceci représente selon les sites jusqu'à 5\% des screening réalisés au cours de l'année 2004. La répartition selon le sexe montre une prédominance du sexe féminin : 111 femmes versus 69 hommes pour le groupe dextropropoxyphène et 69 femmes versus 47 hommes pour le groupe tramadol. Concernant l'âge, la comparaison des moyennes $(\mathrm{m})$ observées $\left(\mathrm{m}_{\text {tramadol }}=45\right.$ ans (écart-type : 20,3 ans) $; m_{D P}=34,6$ ans (écart-type : 18 ans)) basée sur l'écart réduit ( montre que la population correspondant à la mise en évidence de tramadol est significati- vement plus âgée que celle du dextropropoxyphène avec un degré de signification (p) inférieur à $1 \%$ o $\left(\varepsilon_{\text {calculé }}=4,49\right)$.

\section{Les concentrations plasmatiques :}

Les répartitions des concentrations plasmatiques observées sont illustrées par la figure 1 pour le dextropropoxyphène et le norpropoxyphène et par la figure 2 pour le tramadol. Les valeurs seuils choisies correspondent aux concentrations thérapeutiques et potentiellement toxiques du dextropropoxyphène (2) et du tramadol (2-4). Le norpropoxyphène a été dosé dans $57,3 \%$ des cas ; l'étude du rapport [DP]/[NP] montre que dans $87,4 \%$ des cas le norpropoxyphène est retrouvé à une concentration supérieure à celle du dextropropoxyphène. Les métabolites du tramadol n'ont pas été quantifiés mais ils sont le plus souvent présents dans le sang et les urines. La figure 3 présente la répartition des concentrations plasmatiques en paracétamol observées au sein des deux groupes. Concernant les intoxications avec une participation du tramadol, le dosage plasmatique du paracétamol a été réalisé dans seulement 25\% des cas parmi lesquels les résultats obtenus ont traduit une prise de paracétamol dans $48 \%$ des cas (concentration moyenne : 13,8 mg/L, écart type : $24 \mathrm{mg} / \mathrm{L}$ ). Dans le groupe dextropropoxyphène, le dosage du paracétamol a été réalisé dans $73,9 \%$ des cas parmi lesquels il s'est avéré positif dans seulement $65,4 \%$ des cas (concentration moyenne $39,5 \mathrm{mg} / \mathrm{L}$, écart type : $54 \mathrm{mg} / \mathrm{L}$ ). La figure 4 montre qu'il n'y a pas de relation entre les concentrations en dextropropoxyphène ou norpropoxyphène et en paracétamol.

Tableau I : Nombre de cas et méthodes de screening et de dosage du dextropropoxyphène, du tramadol et du paracétamol sur les différents sites.

\begin{tabular}{|c|c|c|c|c|c|c|c|c|c|}
\hline Sites & $\begin{array}{c}\mathbf{N} \\
\text { DP }\end{array}$ & N tramadol & N sang & $\begin{array}{c}\mathbf{N} \\
\text { urines }\end{array}$ & $\begin{array}{c}\text { Total des } \\
\text { screening } \\
\text { sur 2004 }\end{array}$ & $\begin{array}{c}\text { Méthode de } \\
\text { screening }\end{array}$ & Extraction & Estimation & $\begin{array}{c}\text { Méthode de } \\
\text { dosage du } \\
\text { paracétamol }\end{array}$ \\
\hline A & 32 & 15 & 42 & 11 & 812 & REMEDI & on-line & SQ & Enzymatique \\
\hline B & 11 & 6 & 13 & 6 & 902 & REMEDI & on-line & SQ & FPIA \\
\hline C & 1 & 1 & 2 & 0 & 115 & HPLC-DAD & L. alcaline & E & FPIA \\
\hline D & 4 & 4 & 7 & 0 & 630 & HPLC-DAD & L. alcaline & E & FPIA \\
\hline E & 34 & 0 & 34 & 0 & 1404 & HPLC-DAD & L. alcaline & E & Fnzymatique \\
\hline F & 68 & 42 & 73 & 56 & 1350 & REMEDI & on-line & SQ & FPIA \\
\hline G & 30 & 38 & 66 & 4 & 1892 & HPLC-DAD & $\begin{array}{c}\text { L. alcaline +/- } \\
\text { réextraction } \\
\text { acide }\end{array}$ & E & E \\
\hline H & 0 & 7 & 7 & 0 & 1500 & HPLC-DAD & L. alcaline & et FPIA \\
\hline I & 0 & 2 & 2 & 0 & 168 & HPLC-DAD & L. alcaline & E & FPIA \\
\hline J & 0 & 1 & 1 & 0 & NC & LCMS & L. alcaline & E & FPIA \\
\hline K & 0 & 0 & 0 & 0 & NC & REMEDI & on-line & E & FPIA \\
\hline
\end{tabular}

$\mathrm{N}$ : nombre de cas, DP : Dextropropoxyphène, NC : non communiqué, HPLC-DAD : chromatographie liquide haute performance, détection barrette de diodes, LC-MS : chromatographie liquide couplée à la spectrométrie de masse, L : extraction liquide-liquide, SQ : semi-quantitative, E : étalonnage, FPIA : Fluorescence polarization immunoassay. 


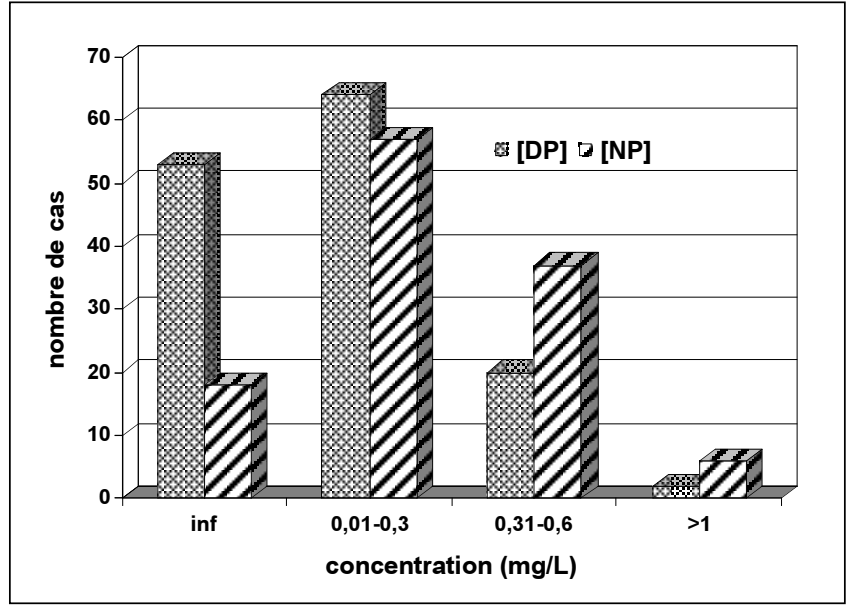

Figure 1 : Répartition des concentrations plasmatiques observées en dextropropoxyphène $(D P)$ et norpropoxyphène $(N P)$.

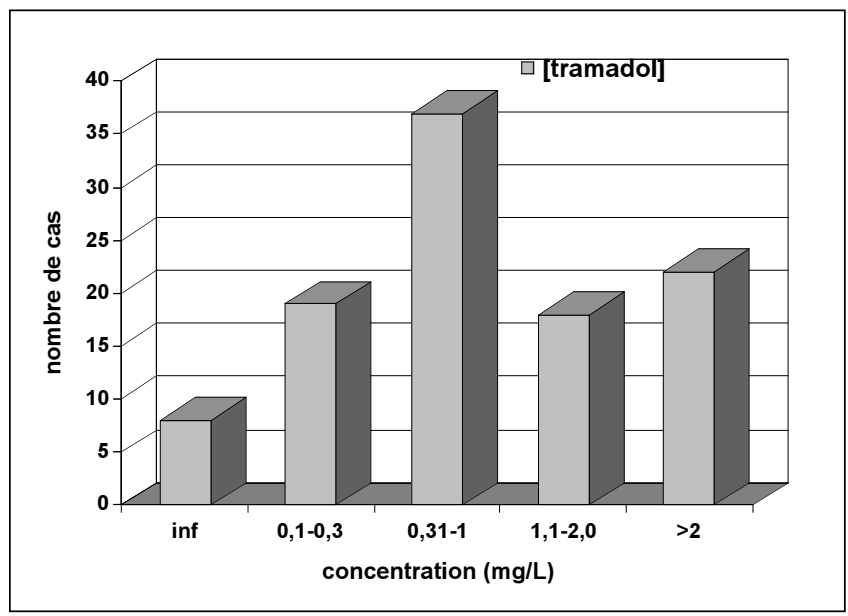

Figure 2 : Répartition des concentrations plasmatiques observées en tramadol.

\section{Associations polymédicamenteuses :}

Nous avons retrouvé un ou plusieurs médicaments associés dans plus de la moitié des cas pour les deux groupes (tableau II). Outre le paracétamol, parmi les

Tableau II : Médicaments retrouvés en association avec le dextropropoxyphène ou le tramadol dans les cas d'intoxications polymédicamenteuses tous sites confondus.

\begin{tabular}{|l|c|c|}
\hline Associations dans & $\begin{array}{c}\text { Dextropropoxyphène } \\
(\mathbf{n = 1 8 0}) \\
\mathbf{6 1 \%} \mathbf{\text { des cas }}\end{array}$ & $\begin{array}{c}\text { Tramadol } \\
(\mathbf{n = 1 1 6}) \\
\mathbf{6 9 \%} \mathbf{\text { des cas }}\end{array}$ \\
\hline Paracétamol & $55 \%$ & $12 \%$ \\
\hline Benzodiazépines & $40 \%$ & $47 \%$ \\
\hline Hypnotiques & $8,3 \%$ & $10 \%$ \\
\hline Antidépresseurs & $8,3 \%$ & $25 \%$ \\
\hline Neuroleptiques & $3,9 \%$ & $13 \%$ \\
\hline Méprobamate & $2,2 \%$ & $1,7 \%$ \\
\hline Cardiotropes & $2,2 \%$ & $2,6 \%$ \\
\hline Alcool & $7,2 \%$ & $3,4 \%$ \\
\hline Autres & $16,7 \%$ & $21 \%$ \\
\hline
\end{tabular}

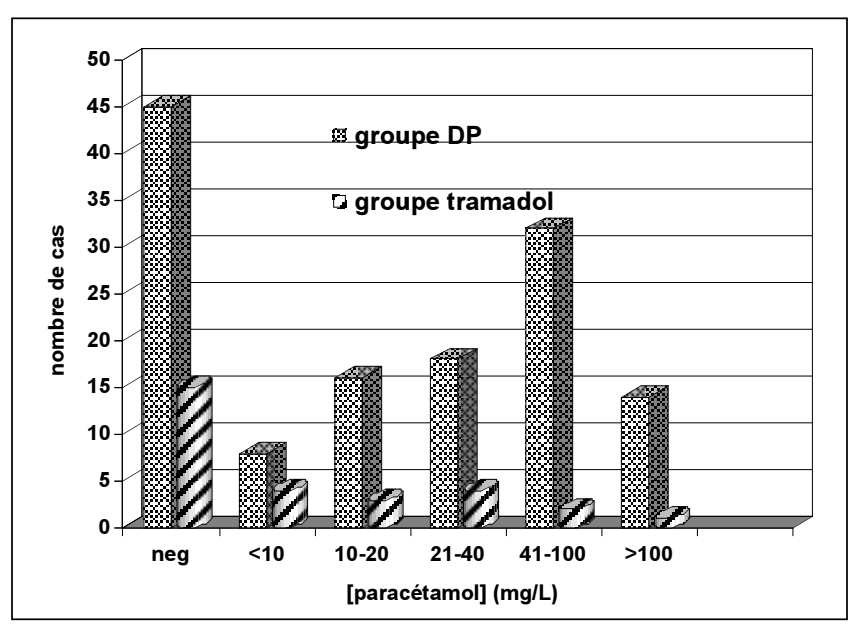

Figure 3 : Répartition des concentrations plasmatiques en paracétamol observées au sein des deux groupes : groupe dextropropoxyphène et groupe tramadol.

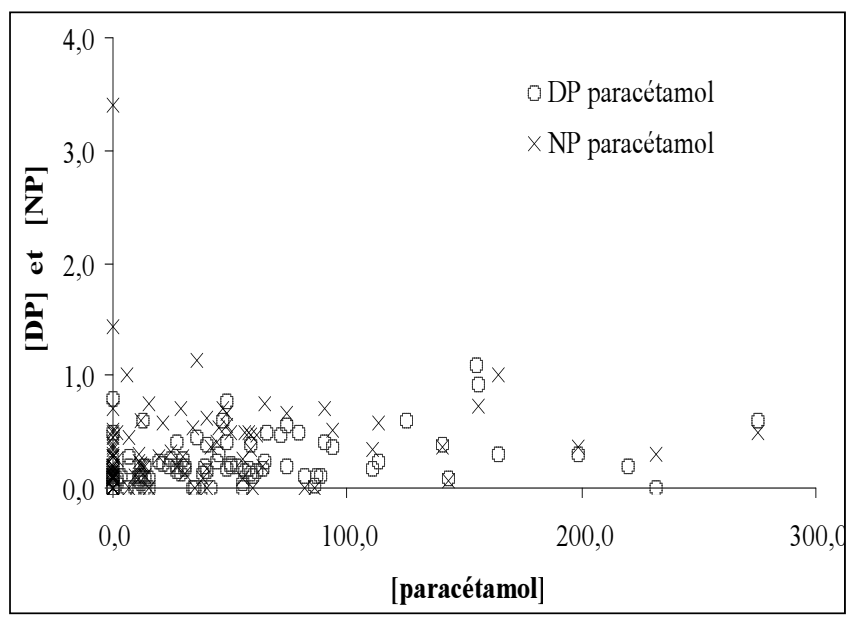

Figure 4 : Réprésentation graphique montrant l'absence de relation entre les concentrations plasmatiques en DP ou NP et paracétamol.

médicaments les plus souvent identifiés ce sont les benzodiazépines qui sont au premier plan suivies par les hypnotiques non benzodiazépiniques (hydroxyzine, zopiclone, zolpidem), les antidépresseurs et les neuroleptiques. Nous avons retrouvé 7 fois l'association dextropropoxyphène-tramadol.

\section{Signes cliniques :}

Le recueil des signes cliniques s'est avéré difficile. Ils n'ont pu être obtenus que dans $57 \%$ des cas pour le groupe dextropropoxyphène et $32 \%$ des cas pour le groupe tramadol. Les fréquences des différentes manifestations cliniques (digestives, cardiaques, respiratoires, neurologiques) sont présentées dans le tableau III. L'analyse des différences de fréquence observées entre les deux groupes a été réalisée par le test statistique du $\chi^{2}$. Nous constatons que les troubles digestifs sont plus fréquents dans le groupe dextropropoxyphè- 
ne, à l'inverse des troubles respiratoires et neurologiques plus souvent observés dans le groupe tramadol et qu'il n'y a pas de différence significative concernant la fréquence d'apparition des troubles cardiaques.

Tableau III : Fréquence des signes cliniques observés dans les deux groupes: groupe dextroproxyphène (DP) et groupe tramadol.

\begin{tabular}{|c|c|c|c|}
\hline $\begin{array}{c}\text { Pourcentage de recueil } \\
N\end{array}$ & DP & Tramadol & \\
\hline $\begin{array}{c}\text { Digestifs } \\
\text { Despiratoires } \\
\text { nausées, vomissements, douleurs abdominales }\end{array}$ & $27 \%$ & $8 \%$ & $\mathrm{p}<0,02^{*}$ \\
\hline $\begin{array}{c}\text { Répression ou dyspnée } \\
\text { Cardiaques }\end{array}$ & $7,8 \%$ & $29,7 \%$ & $\mathrm{p}<0,001^{*}$ \\
\hline $\begin{array}{c}\text { Neurologiques } \\
\text { troubles du rythme ou de la conduction, état de choc }\end{array}$ & $9,7 \%$ & $10,8 \%$ & $\mathrm{NS}^{* *}$ \\
\hline $\begin{array}{c}\text { convulsions, somnolence, coma, agitation, } \\
\text { tremblements, confusion, troubles de l'équilibre, } \\
\text { céphalées, myosis, absences }\end{array}$ & $46 \%$ & $89 \%$ & $\mathrm{p}<0,001^{*}$ \\
\hline
\end{tabular}

*test du $\chi^{2}-* *$ non significatif - DP : dextropropoxyphène

\section{Discussion}

Cette étude réalisée sur plusieurs sites présente les inconvénients d'une étude multicentrique : non standardisation des protocoles et des méthodes analytiques, certains sites n'ont rapporté que les cas de dextropropoxyphène et d'autres que les cas de tramadol. Le nombre d'intoxications relevées avec une participation du dextropropoxyphène ou du tramadol représente un faible pourcentage sur l'ensemble des screening toxicologiques réalisés sur les différents sites : moins de $5 \%$. Le tramadol est prescrit en $2^{\text {ème }}$ intention dans le traitement de la douleur après épuisement de l'efficacité de l'association dextropropoxyphène-paracétamol ce qui explique la différence de répartition en fonction de l'âge observé entre les deux groupes. Dans certains pays comme la Suède, les intoxications au dextropropoxyphène sont fréquentes $(27 \%$ des intoxications mortelles) avec des concentrations plasmatiques en dextropropoxyphène retrouvées supérieures aux concentrations thérapeutiques. Elles touchent en général une population relativement jeune (moyenne d'âge 43 ans) chez laquelle on retrouve l'association dextropropoxyphène alcool $(5,6)$. Dans notre étude, les concentrations plasmatiques observées en dextropropoxyphène et en norpropoxyphène sont faibles et le plus souvent comprises dans la zone thérapeutique. Ainsi la concentration moyenne observée en dextropropoxyphène est égale à $0,17 \mathrm{mg} / \mathrm{L}$ (écart type : $0,27 \mathrm{mg} / \mathrm{L}$ ) pour des valeurs thérapeutiques comprises entre 0,05 et $0,3 \mathrm{mg} / \mathrm{L}(2)$; celle en norpropoxyphène est égale à $0,45 \mathrm{mg} / \mathrm{L}$ (écart type : $1,33 \mathrm{mg} / \mathrm{L}$ ) pour des valeurs considérées comme toxiques au-delà de $3 \mathrm{mg} / \mathrm{L}$ (7). Seulement deux cas présentent une concentration plasmatique en dextropropoxyphène supérieure à la concentration toxique de $1 \mathrm{mg} / \mathrm{L}$ (2). La littérature rapporte que la concentration plasmatique en norpropoxyphène est nettement supérieure à la concentration plasmatique en dextropropoxyphène même si le prélèvement est précoce (8). Ceci se vérifie dans notre étude à l'exception de 13 cas pour lesquels la valeur du rapport $[\mathrm{DP}] /[\mathrm{NP}]$ est supérieur à 1 . Cependant, nous n'avons pas tenu compte du délai écoulé entre les prélèvements et la prise médicamenteuse, délai le plus souvent inconnu dans les cas d'intoxication et de toute façon non maîtrisable. Les sites qui ont répondu pour le dextropropoxyphène sont des sites français. En France, le dextropropoxyphène est toujours associé au paracétamol quelque soit la spécialité pharmaceutique. Compte tenu des proportions des deux molécules dans ces associations (27 ou $30 \mathrm{mg}$ de dextropropoxyphène pour $400 \mathrm{mg}$ de paracétamol), l'intoxication au paracétamol est toujours au premier plan en terme de gravité (1). Il est fréquent de retrouver encore du dextropropoxyphène ou de ses métabolites dans le sang alors qu'il n'y a plus de paracétamol du fait des demi-vies d'élimination plasmatique très différentes de ces deux molécules. La présence de dextropropoxyphène ou de norpropoxyphène signe alors obligatoirement une prise de paracétamol. Pour le tramadol, les valeurs des taux thérapeutiques décrites dans la littérature sont très variables : de 0,1 à $0,3 \mathrm{mg} / \mathrm{L}$ (3), 0,28 à $0,61 \mathrm{mg} / \mathrm{L}$ (4) ou jusqu'à $1 \mathrm{mg} / \mathrm{L}$ (7). Les concentrations plasmatiques retrouvées lors d'intoxications mortelles varient de 0,03 à $134 \mathrm{mg} / \mathrm{L}$. La gravité de ces intoxications est plus souvent liée aux associations médicamenteuses (benzodiazépines, antidépresseurs tricycliques et inhibiteurs de la recapture de la sérotonine) qu' au tramadol seul $(4,9$, 10) avec une potentialisation de la toxicité liée à la composante monoaminergique du tramadol. Seulement deux cas d'intoxications mortelles au tramadol ont été rapportés $(3,11)$. Dans notre étude, nous n'avons relevé aucune intoxication majeure au tramadol et les concentrations plasmatiques sont généralement plus élevées que les concentrations thérapeutiques classiquement admises: la concentration moyenne observée est égale à $1,39 \mathrm{mg} / \mathrm{L}$ (écart type : $1,47 \mathrm{mg} / \mathrm{L}$ ). Nous avons répertorié 22 concentrations en tramadol supérieures à $2 \mathrm{mg} / \mathrm{L}$ avec une concentration maximale à 7,3 mg/L, cas pour lesquels les signes cliniques ne sont pas évocateurs d'une éventuelle intoxication au tramadol. Peu de dosages de paracétamol ont été réalisés dans le groupe tramadol et ils se révèlent positifs seulement dans 14 cas : le tramadol n'est pas forcément associé au paracétamol. Il n'est associé au paracétamol que dans deux spécialités récentes : le Zaldiar $^{\circledR}$ et l'Ixprim $^{\circledast}$. Concernant les médicaments associés retrouvés, tous sites confondus nous constatons que le tramadol est plus souvent associé aux antidépresseurs et aux 
neuroleptiques que le dextropropoxyphène. Il s'agit là d'un biais de recrutement ; certains sites n'ont répondu que pour le dextropropoxyphène, d'autres que pour le tramadol, or tous les sites n'ont pas la même sensibilité dans la détection des médicaments associés et si l'on ne considère que les sites ayant répondu à la fois pour le dextropropoxyphène et pour le tramadol nous n'observons plus de différence entre les deux groupes. Les signes cliniques n'ont pu être recueillis pour tous les patients. Les troubles digestifs, plus fréquemment observés dans la population dextropropoxyphène, sont certainement liés à l'ingestion concomitante obligatoire de paracétamol dans ce groupe. Les troubles respiratoires significativement plus fréquents dans la population tramadol ne représentent cependant que 11 cas sur 37 dont 8 dépressions respiratoires. Ce sont les troubles neurologiques qui sont au premier plan : la prédominance de la somnolence est certainement liée au grand nombre de benzodiazépines retrouvées en association; les convulsions sont plus fréquentes dans le groupe tramadol mais ne représentent que 4 cas sur 37 avec 2 cas pour lesquels la concentration plasmatique en tramadol est inférieure à la concentration toxique de $1 \mathrm{mg} / \mathrm{L}$ mais 2 cas avec une concentration en tramadol supérieure à $2 \mathrm{mg} / \mathrm{L}$ (Figure 5). Ces troubles neurologiques plus fréquents avec le tramadol pourraient être liés à son action inhibitrice de la recapture des monoamines (noradrénaline et sérotonine). Cependant, nous ne constatons pas de lien entre l'apparition des troubles neurologiques et une association médicamenteuse pouvant favoriser un syndrome sérotoninergique. Les comas sont observés dans plus de $10 \%$ des cas dans les 2 groupes. Pour le groupe dextropropoxyphène, les 14 cas de comas correspondent à des intoxications polymédicamenteuses chez lesquelles les concentrations retrouvées en dextropropoxyphène et norpropoxyphène sont faibles ([DP] $<0,4 \mathrm{mg} / \mathrm{L},[\mathrm{NP}]<0,7 \mathrm{mg} / \mathrm{L}$ ) et peu différentes des valeurs observées sur l'ensemble de la population. Ces comas sont donc plutôt le fait des associations que du dextropropoxyphène lui même. Parmi les 8 comas pour lesquels nous avons retrouvé du tramadol, 2 cas correspondent à des maladies neurologiques en fin de vie, 5 à des intoxications polymédicamenteuses et il y a 1 cas pour lequel seul du tramadol a été mis en évidence mais à une concentration faible $(0,36 \mathrm{mg} / \mathrm{L})$. Cependant, le O-desméthyl tramadol (métabolite actif) n'a pas été quantifié, nous ignorons le délai de prise en charge du patient et nous ne pouvons exclure l'hypothèse d'une intoxication par une substance non identifiée par le système chromatographique utilisé. Parmi ces comas, seulement 3 patients présentent une concentration en tramadol supérieure à $2 \mathrm{mg} / \mathrm{L}$ par rapport aux 22 cas observés sur l'ensemble de la population. Il parait donc difficile d'imputer ces comas au tramadol seul.

En résumé, dans cette étude nous n'avons mis en évidence aucune relation entre les concentrations plasmatiques en dextropropoxyphène ou en tramadol et la présence de l'un ou l'autre des signes cliniques qu'il s'agisse des comas, des dépressions respiratoires, des troubles cardiaques ou des convulsions et la comparaison des 22 cas présentant des concentrations en tramadol supérieures à $2 \mathrm{mg} / \mathrm{L}$ avec ceux présentant une concentration plus faible ne montre pas de différence au niveau de l'intensité des signes cliniques ni de la gravité.

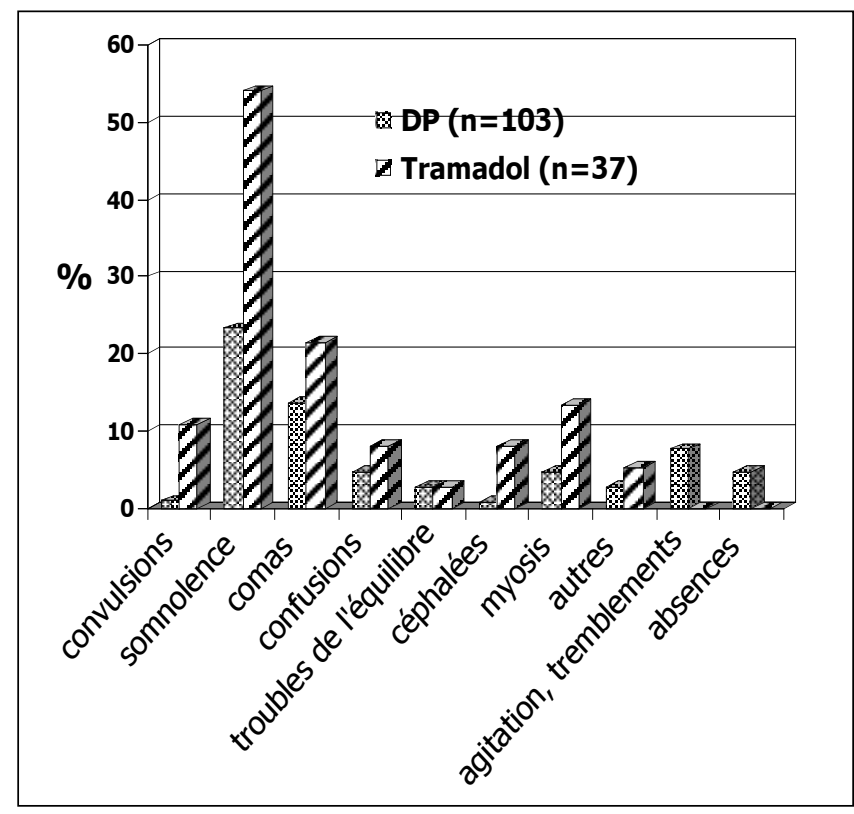

Figure 5 : Fréquences des signes neurologiques observés dans les deux groupes : dextropropoxyphène et tramadol.

\section{Conclusion}

Dans le groupe correspondant à une prise de dextropropoxyphène, nous n'observons aucune intoxication majeure au dextropropoxyphène, aucun signe de gravité n'est dans cette étude à rapporter directement au dextropropoxyphène ou au norpropoxyphène mais c'est l'intoxication au paracétamol qui est au premier plan. La mise en évidence de dextropropoxyphène ou de norpropoxyphène dans le sang ou les urines peut donc permettre un diagnostic tardif d'intoxication au paracétamol. Dans le groupe de patients pour lesquels du tramadol a été retrouvé avec des concentrations plasmatiques parfois élevées (supérieures à $2 \mathrm{mg} / \mathrm{L}$ dans 22 cas), nous n'avons pas observé au cours de l'étude de relation entre la gravité et les concentrations plasmatiques. A priori, il découle de cette étude que les concentrations toxiques en tramadol sont très supérieures à la concentration toxique de $1 \mathrm{mg} / \mathrm{L}$. 


\section{Références}

1. AFSSAPS Analyse des ventes de médicaments aux officines et aux hôpitaux en France 1993-2003, $5^{\text {ème }}$ édition 2005.

2. Schulz M., Schmoldt A. Therapeutic and toxic blood concentrations of more than 800 drugs and other xenobiotics. Pharmazie $2003 ; 58: 447-74$.

3. Musshoff F., Madea B. Fatality due to ingestion of tramadol alone. Forensic Sci. Int. 2001; 116 : 197-9.

4. Clarkson J.E., Lacy J.M., Fligner C.L., Tiersch N., Howard J., Harruff R.C., Logan B.K. Tramadol (Ultram) concentrations in death investigation and impaired driving cases and their significance. J. Forensic Sci. 2004 ; 49 : $1101-5$.

5. Jonasson B., Jonasson U., Saldeen T. Among fatal poisonings dextropropoxyphène predominates in younger people, antidepressants in the middle aged and sedatives in the elderly. J. Forensic Sci. $2000 ; 45: 5-10$.

6. Jonsson A., Holmgren P., Ahlner J. Fatal intoxications in a Swedish forensic autopsy material during 1992-2002. Forensic Sci. Int. 2004 ; 143 : 53-9.

7. Flanagan R.J. Guidelines for the interpretation of analytical toxicology results and unit of measurement conversion factors. Ann. Clin. Biochem. 1998 ; 35 : 261-7.

8. Gustafson A., Gustafson B. Acute poisoning with dextropropoxyphene. Clinical symptoms and plasma concentrations. Acta Med. Scand. 1976 ; $200: 241-8$.

9. Levine B., Ramcharitar V., Smialek J.E. Tramadol distribution in four post-mortem cases. Forensic Sci. Int. 1997 ; $86: 43-8$.

10. Michaud K., Augsburger M., Romain N., Giroud C., Mangin P. Fatal overdose of tramadol and alprazolam. Forensic Sci. Int. 1999; 105 : 185-9.

11. Loughrey M.B., Loughrey C.M., Johnston S., O'Rourke D. Fatal hepatic failure following accidental tramadol overdose. Forensic Sci. Int. 2003 ; 134 : 232-3. 